INRA Prod. Anim.,

2007, 20 (2), 137-150

\title{
Recommandations alimentaires pour les vaches de réforme à l'engrais. Développement d'un modèle d'estimation de la composition du gain de poids et des besoins associés
}

F. GARCIA, J. AGABRIEL

INRA, UR1213 Herbivores, F-63122 Saint-Genès Champanelle, France Courriel :florence.garcia@clermont.inra.fr

\begin{abstract}
Dans les différents pays de l'Union Européenne la production de viande issue des femelles (vaches adultes et génisses) représente de 32 à $88 \%$ des gros bovins abattus (Cabaraux et al 2005), ce qui correspond environ à un tiers de la consommation totale de viande bovine en Europe. Dans cet ensemble, la France est le pays où l'on consomme le plus de viande issue de

également associés à des itinéraires de finition variés, avec des rations de concentration énergétique différente, car l'engraissement peut se faire à l'auge, avec des régimes à base de foin, à base d'ensilage de maïs ou encore avec de fortes proportions de concentrés. Mais il peut aussi se faire au pâturage où les moyens d'action de l'éleveur sur la ration sont alors plus limités.
\end{abstract} vaches en provenance des deux troupeaux, laitier et allaitant. Au niveau des exploitations, les recettes apportées par la vente des femelles de réforme engraissées représentent une part importante du produit bovin, estimée en moyenne à $10 \%$ dans les élevages laitiers (Veysset comm. pers., données 2004-2005) et à près de $30 \%$ dans les élevages allaitants. Dans ces derniers, cette proportion varie selon les régions et les races et passe de $17 \%$ en troupeau Salers, à $26 \%$ et $30 \%$ en Charolais et Limousin (Lherm comm. pers., données 2002-2005). Les poids et cotation différents des deux types de vaches en fonction de leur conformation en sont la principale cause $(3,38 € / \mathrm{kg}$ net pour une vache de note d'engraissement 3 et une conformation notée R, charolaises de $400 \mathrm{~kg}$ carcasse par exemple, $2,38 € / \mathrm{kg}$ net pour une carcasse de vache laitière de $350 \mathrm{~kg}$ classée O, Données Ofival septembre 2006). Les observations des réseaux d'élevage rapportent la grande variabilité phénotypique des vaches de réforme au sein d'une même race, engraissées ou non (Bastien et Brouard-Jabet 2000, Pierret et al 2002, 2004). En effet, l'âge, le format, l'état sanitaire, l'état physiologique (vide ou en gestation, tarie ou en lactation) et l'état d'engraissement sont très variables au moment de la réforme, et constituent autant de facteurs modifiant leur aptitude à l'engraissement. Ces animaux sont
Cette très grande variabilité des animaux se traduit également par une certaine inconstance des qualités organoleptiques des viandes produites. Les animaux âgés (10 ans et plus) produisent en général une viande de tendreté moindre (Malterre et Jones 1992) mais aussi une viande souvent plus riche en lipides intramusculaires, caractérisée par une flaveur et une jutosité élevées (Dransfield et al 2003). Ces résultats ne sont pas toujours aussi marqués. Ainsi, Bastien et al (2002) n'ont pas mesuré d'effet de l'âge sur les notes de tendreté de viandes issues du muscle longissimus dorsi ou du muscle supra spinatus chez des vaches jeunes ou âgées de race Normande (5,9 vs 6,4 sur 10 pour longissimus dorsi) ou Limousine (7,0 vs 7,0 sur 10 pour longissimus dorsi).

Par des pratiques d'élevage diverses, le niveau énergétique de la ration et la nature de l'alimentation, les éleveurs peuvent intervenir pour moduler à la fois la composition tissulaire de la carcasse, la proportion moyenne de lipides intramusculaires et la couleur de la viande produite. Les recommandations alimentaires établies en 1988 (UF, PDI, UE, Geay et Micol 1988) pour les vaches de réforme à l'engrais tiennent compte du poids vif, du gain de poids vif et du type d'animal (laitier ou allaitant) considéré. Elles ont été construites sur la base des observations expérimen- tales disponibles en considérant globalement la relation entre le gain de poids et les apports nutritionnels sans distinguer la part des besoins associée à l'entretien de la part associée au gain de poids (par opposition à la méthode factorielle utilisée pour les autres animaux en croissance et à l'engrais, Geay et al 1987). Ces recommandations ne permettent pas de prendre en compte tous les facteurs d'influence, l'état d'engraissement ou encore l'âge des animaux. Dans le cadre de la réactualisation des recommandations alimentaires (Garcia et al 2007a), il est donc apparu nécessaire de proposer un nouveau modèle de prédiction des besoins énergétiques des vaches de réforme à l'engrais prenant en considération toutes les informations disponibles dans les exploitations d'élevage.

Notre démarche globale a consisté à étendre l'approche développée par Robelin et Daenicke (1980) et à utiliser la méthode factorielle qui ajoute aux besoins d'entretien de l'animal (métabolisme digestif, renouvellement cellulaire, homéothermie...), les besoins associés au gain de poids, somme de l'énergie contenue dans le gain de lipides et le gain de protéines (Vermorel et al 1987).

Nous rappellerons rapidement dans cet article, les modèles de composition corporelle et de composition du gain de poids chez des bovins adultes développés dans la littérature en rappelant initialement qu'un tel travail avait été proposé par Hoch et al (2004) pour les modèles d'animaux en croissance. Nous préciserons les forces motrices biologiques qui nous ont guidé avant de décrire ensuite précisément le modèle d'engraissement que nous appelons VDR, ses principes et les équations associées. La dernière partie de cet arti- 
cle concernera le paramétrage et la validation du modèle ainsi que son application pour établir les nouvelles recommandations alimentaires en UF des vaches de réforme à l'engrais, sans préciser ici la détermination des besoins azotés (PDI), ni celle de la capacité d'ingestion (UE).

\section{1 / Reprise de poids et reprise d'état. Caractéris- tiques de l'engraissement des vaches}

L'objet de cette synthèse n'est pas de décrire exhaustivement tous les phénomènes biologiques qui modifient la composition corporelle d'un animal adulte, mais de rappeler les principaux processus que nous avons essayé de transcrire dans notre démarche de modélisation.

Les études sur l'engraissement des vaches de réforme de race à viande ont été réalisées majoritairement dans les années 80-90 en France (Malterre et al 1989, Robelin et al 1990, Agabriel et al 1991, Roux et al 1993, Dumont et al 1997), au Danemark (Shemeis et al 1994) ou plus récemment en Belgique (Cabaraux et al 2004, 2005, Fiems et al 2005). Elles représentent la grande majorité des observations disponibles sur cette production, et ces recommandations sont construites principalement à partir de ces données.

Les données sur vaches laitières sont les plus anciennes, mais les lois biologiques qu'elles sous-tendent restent bien entendu valables malgré l'augmentation du format des vaches. Cette augmentation est de l'ordre de $+30 \mathrm{~kg}$ et $+50 \mathrm{~kg}$ sur les poids carcasse de vaches notées 3,0 (290 à $320 \mathrm{~kg}$ poids carcasse en race Prim Holstein ou Montbéliarde, 290 à $350 \mathrm{~kg}$ en race Normande ; Malterre et Jones 1992, Meffe et al 2005). En considérant que les différents constituants du corps évoluent de manière homothétique à l'accroissement du format, ces études restent donc encore largement utilisables, car les biais que cette hypothèse peut induire restent modestes.

Les études descriptives d'évolution de l'état de vaches à viandes (Jenkins et Ferrell 1997) ou laitières permettent de comprendre les cinétiques relatives des dépôts de tissus. Les variations de poids, de masse corporelle des vaches adultes et l'amplitude des variations des composants tissulaires ou chi- miques ont en effet été analysées dans plusieurs expériences faites sur des génotypes variés, du plus laitier (Prim Holstein) au plus à viande (Blanc Bleu Belge (BBB)). Les méthodes utilisées dans les expérimentations sont soit l'abattage de vaches choisies en état très variable (Wright et Russel 1984b, Fiems et al 2005) soit l'abattage de lots appariés maigres puis engraissés sur des durées variables (Robelin et al 1990). La composition corporelle est estimée dans les données françaises par la méthode du diamètre des adipocytes du tissu adipeux sous cutané caudal (Robelin et Agabriel 1986). L'estimateur pratique de l'état d'engraissement est dans toutes ces expériences la note d'état corporel mesurée sur une échelle allant de 0 (très maigre) à 5 (très gras) (Bazin 1984, pour les vaches laitières, Agabriel et al 1986 pour les races à viande).

$\mathrm{Au}$ cours de l'engraissement, le rendement carcasse augmente (Malterre et Jones 1992), et la part des viscères diminue. Les muscles squelettiques représentent environ 65 à $70 \%$ de la carcasse chez les vaches de race à viande et leur évolution quantitative est modeste, de l'ordre de 20 à $25 \mathrm{~kg}$ au cours de l'engraissement, et jusqu'à 35$45 \mathrm{~kg}$ chez des vaches de réforme Blanc Bleu Belge culardes (Cabaraux et al 2003, 2004). Cette évolution est peu modifiée par le niveau d'apport alimentaire même pour les races qui présentent un fort développement musculaire comme la Blanc Bleu Belge (Cabaraux et al 2004). Dans les viscères, la part des dépôts adipeux augmente proportionnellement plus rapidement que la part des dépôts adipeux dans la carcasse (Robelin et al 1990, Jenkins et Ferrell 1997). Le gras intramusculaire est le plus tardif à se déposer (persillé) mais les résultats disponibles ne sont encore pas assez complets pour que l'on puisse en proposer un modèle prédictif couvrant plusieurs types de situations (Hoch et al 2002). Il faut aussi rappeler, car cela a des implications pratiques importantes, que les dépôts adipeux sous-cutanés ont l'accroissement relatif le plus élevé par rapport aux dépôts totaux, de l'ordre d'un facteur $7 \mathrm{chez}$ les vaches Charolaises par exemple. Cette modification importante est aisément appréciée par la manipulation et se traduit en variation de note d'état sans erreur majeure. Un point de note correspond ainsi en moyenne à $30 \mathrm{~kg}$ de lipides (25 à $35 \mathrm{~kg}$ selon les expérimentations : $31 \mathrm{~kg}$ de lipides par exemple pour des vaches BBB (Fiems et al 2005), 27 pour des vaches laitières (Remond et al 1988)) et entre 40 et $45 \mathrm{~kg}$ de masse corporelle.

Pour un régime donné, la reprise journalière de poids vif observée dans des expérimentations d'engraissement varie en fonction de la durée d'engraissement. Elle suit une évolution curvilinéaire, rapide au début de l'engraissement (jusqu'à $45 \mathrm{j}$ à $55 \mathrm{j}$ avec des rations concentrées en énergie) et diminue ensuite de manière plus ou moins prononcée selon le niveau des apports et l'état de la vache (Malterre 1986, Agabriel et al 1991, Dumont et al 1991, par exemple).

Les raisons en sont multiples, et la revue récente de Cabaraux et al (2005) a rappelé les grands principes qui affectent et contrôlent biologiquement la reprise d'état des vaches adultes :

- la composition du dépôt est de plus en plus riche en lipides et pauvre en protéines et en eau. Le dépôt devient donc de plus en plus coûteux en énergie $(9,37 \mathrm{kcal} / \mathrm{kg}$ de lipides vs $5,48 \mathrm{kcal} / \mathrm{kg}$ de protéines). Parallèlement, l'efficacité d'utilisation de l'énergie métabolisable disponible diminue. Les compositions des gains de poids ont été reconstituées à partir d'observations d'abattages expérimentaux (Wooten et al 1979, Wright et Russel 1984b, Malterre 1986, Agabriel et al 1991, exemples figure 1). La proportion moyenne de tissus adipeux ou de lipides dans le gain de masse corporelle peut cependant varier fortement d'environ $30 \%$ à $90 \%$ selon les études, les conditions et les génotypes. Mesurés par exemple entre lots de vaches taries Charolaises notées 1 à 4, les lipides représentent $74 \%(97 \mathrm{~kg})$ du gain de masse corporelle $(130 \mathrm{~kg})$ et sont contenus principalement dans les dépôts adipeux $(87 \%)$. Cette proportion moyenne se nuance selon les génotypes. Pour les plus naturellement maigres comme la BBB (Fiems et al 2005), un gain de $188 \mathrm{~kg}$ de masse corporelle entre 1 et 4 correspond à $35 \%$ de gain de lipides $(64 \mathrm{~kg})$, et à l'inverse chez des Holstein le gain de dépôts adipeux correspond à $78 \%$ du gain de masse corporelle (87 pour $114 \mathrm{~kg}$ ) (Robelin et al 1990). Mais la part des lipides dans le gain augmente également régulièrement au cours de l'engraissement. Pour les vaches $\mathrm{BBB}$ par exemple elle est respectivement de $25,6 \%$ et $44,5 \%$ dans les intervalles $450 / 500 \mathrm{~kg}$ ou $800 / 850 \mathrm{~kg}$ de masse corporelle, et chez les Charolaises elle passe également de près de $40 \%$ entre les notes 1 et 2,5 pour une masse corporelle variant entre 513 à $616 \mathrm{~kg}$, à près de 
Figure 1. Proportion de lipides dans la variation de poids vif vide selon l'état de la masse corporelle (lipides dans le poids vif vide).

Proportion de lipides dans la variation de poids vif vide

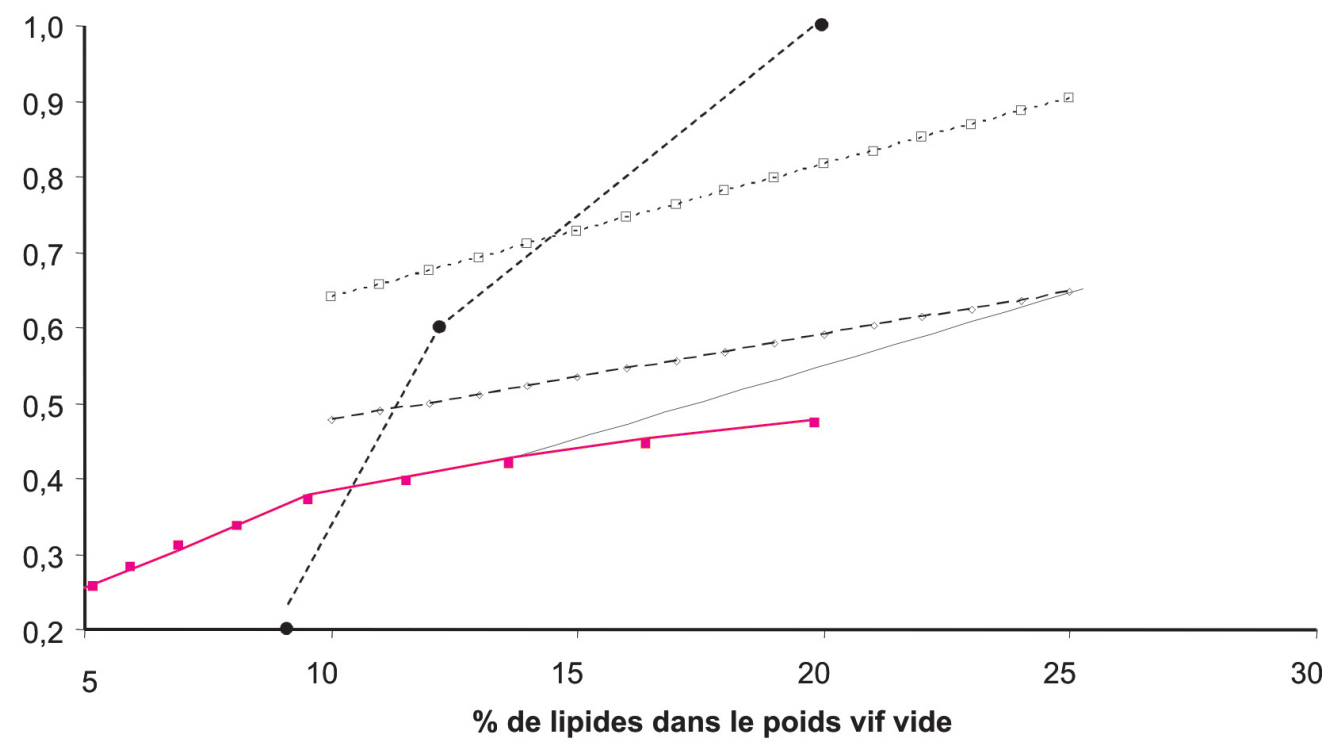

Sources

$\begin{array}{lll}\text { Williams et Jenkins } 1997 & \ldots \text { INRA } 88 \\ \ldots . . . . & \text { Wright etRussel 1984, Charolais } \longrightarrow \text { - Fiems et al } 2005\end{array}$

$100 \%$ entre 616 et $643 \mathrm{~kg}$ (Agabriel et Petit 1987);

- le besoin d'entretien augmente régulièrement sous l'effet de l'accroissement de la masse corporelle. Les résultats de la littérature ont montré que les besoins d'entretien sont liés au poids métabolique de l'animal et aussi en grande partie au poids des viscères et du foie en particulier (Chilliard et al 1995). La masse protéique a un turnover important et donc un besoin d'entretien supérieur à la masse lipidique, mais l'augmentation de l'état d'engraissement des animaux diminue le rendement énergétique du dépôt. Le mode de calcul (régression pour une variation de poids nulle) peut affecter cette baisse au besoin d'entretien, et on observe alors que les vaches maigres ont moins de besoins d'entretien que des grasses. Ainsi, les observations de la littérature ne donnent pas de résultats clairs quant à l'effet de l'état d'engraissement sur le besoin d'entretien (Ortigues et al 1993, Birnie et al 2000, Houghton et al 1990) ;

- la capacité d'ingestion mesurée en UEB (ou l'appétit mesuré en MS ingérée par $\mathrm{kg}$ de gain) diminue lorsque l'état d'engraissement augmente (- 0,5 UEB par point de note sur des génisses ou des vaches laitières (Hoch et al 2005, Faverdin et al 2007) et jusqu'à 2 UEB par point sur des vaches Charolaises taries, (Agabriel et Lassalas 2001). Ce processus est cependant soumis à une forte variabilité individuelle.
Ces trois principes se combinent de manière plus ou moins accentuée selon la race de la vache et sa précocité, son âge et l'état initial au démarrage de la phase d'engraissement.

Il en découle que l'état d'engraissement initial est un déterminant de la prise de poids et de sa composition au cours de l'engraissement. Une vache maigre en début d'engraissement aura une reprise de poids rapide, grâce à une capacité d'ingestion élevée, un gain de poids riche en eau et une amélioration du rendement d'utilisation de l'énergie métabolisable. Un animal présentant une note d'état corporel élevée à la réforme réalisera un gain de poids vif moindre, avec une efficacité alimentaire réduite.

Les principaux facteurs de variation qui affectent l'aptitude à la reprise de poids et la composition de cette reprise (race, âge physiologique, GMQ, alimentation : nature, type de régime et composition, rapport énergie/azote) ont également été décrits dans la revue de Cabaraux et al (2005). Nous les citons ici pour mémoire sans en décrire finement l'importance.

Ainsi l'effet du niveau nutritionnel est certainement le levier le plus simple dont l'éleveur dispose pour maîtriser le dépôt de gras et atteindre l'optimum demandé par le marché. Les recommandations alimentaires ont pour objectif d'aider à la gestion de l'engraissement pour arriver à une reprise de poids qui serait idéale.

Wright et Russel 1984, Angus

\section{2 / Revue des modèles de la reprise ou de la variation d'état chez la vache adulte}

Si les évolutions des réserves chez les vaches taries ou en production sont connues (Trigg et Topps 1981, Wright et Russell 1984a, Chilliard 1987, Chilliard et al 1991, Jenkins et Ferell 1997), la modélisation de la variation du gain de poids et de composition corporelle chez les vaches adultes est plus rare. Elle concerne principalement les vaches en production et, le plus souvent dans la littérature, les vaches laitières traites.

On distingue deux types de modèles : les modèles empiriques qui sont descriptifs par opposition aux modèles mécanistes plus explicatifs. Les modèles empiriques sont en général obtenus par l'ajustement de données expérimentales (Sauvant 1992), alors que les modèles mécanistes reposent sur la représentation des phénomènes à des niveaux sous-jacents (par exemple évolution de la composition corporelle pour prédire le poids vif de l'animal).

Certains modèles visent à prédire les variations de composition selon le stade physiologique de la vache (Williams et Jenkins 1997, 2003a, b). Ils se focalisent le plus souvent sur le début de la lactation notamment pour les vaches laitières à haut potentiel, au 
moment où la mobilisation lipidique est la plus intense (Roguet et Faverdin 1999). Puis ils estiment le besoin à l'échelle de la lactation entière. D'autres, plus empiriques et globaux, intègrent cette estimation comme sous modèles dans des ensembles plus vastes centrés sur la dynamique du troupeau ou l'exploitation d'élevage (Sanders et Cartwright 1979a, b, Hirooka et al 1998, Tess et Kolstad 2000). Néanmoins, dans la plupart des cas, par le biais de la variation de la composition corporelle, ces modèles estiment toujours les besoins énergétiques des animaux. Ils reposent généralement sur les principes suivants :

- la définition d'un animal référence évoluant au cours du temps, voire d'une courbe de croissance de référence ;

l'utilisation d'une approche «énergétique» qui considère l'énergie métabolisable ingérée et sa répartition en énergie nécessaire pour l'entretien, la gestation, la lactation et la croissance ;

- la prise en compte des processus biologiques, plus ou moins simplifiés selon les hypothèses retenues et leurs transcriptions mathématiques sous forme de lois de réponse en posant les équations correspondantes nécessaires.

Les modèles recensés utilisent souvent une référence, qui peut être un animal «moyen» à maturité (Sanders et Cartwright 1979a, b, Bruce et al 1984, Williams et Jenkins 1997, 2003a, b) voire la courbe de croissance d'un animal «moyen» (Sanders et Cartwright 1979a, b, Hirooka et al 1998, Tess et Kolstad 2000). La vache standard de référence à maturité est ainsi caractérisée par son poids vif, et la composition en tissu adipeux de sa masse corporelle. Dans la littérature anglo-saxonne, il est couramment admis que cette proportion est de $25 \%$ et cela quelle que soit la race (Sanders et Cartwright 1979 a, b, Williams et Jenkins 1997) ce qui parait élevé pour les animaux de nos races à viande françaises, pour lesquels ce chiffre est plus proche de $18 \%$. Le modèle développé par Williams et Jenkins (1997) estime la composition des variations de poids vif vide chez les vaches adultes taries. La variation de poids vif vide et la variation du poids de lipides sont liées comme dans le modèle des animaux en croissance de Robelin et Daenicke (1980). Ce modèle dispose ensuite de modulations pour la vache en début de lactation, la vache soumise à un déficit énergétique prolongé et la vache qui reprend de l'état corporel en pleine lactation.
Une courbe de croissance de référence peut être utilisée avec une approche décomposant l'utilisation de l'énergie métabolisable, comme chez Bruce et al (1984). Ces auteurs définissent ainsi un potentiel de croissance chez les vaches selon une équation de Gompertz où le taux de croissance dépend du poids à maturité. Sanders et Cartwright (1979b), Hirooka et al (1998) et Tess et Kolstad (2000) définissent la courbe de croissance de référence selon trois points clés : la naissance, le sevrage ou un an d'âge, et enfin la maturité suivant en cela les lois de Brody (1945). La croissance entre la naissance et le sevrage ou un an d'âge est alors supposée linéaire tandis que la croissance audelà d'un an, et donc pour les vaches, suit une loi de Brody (1945). Pour Sanders et Cartwright (1979b) et Hirooka et al (1998), le poids vif est estimé selon cette courbe de croissance, tandis que pour Tess et Kolstad (2000), il s'agit de la masse délipidée. Des pourcentages de lipides et de contenu digestif sont définis aux trois points clés et permettent de reconstituer la composition corporelle de l'animal dans ces trois modèles. Sanders et Cartwright (1979b) n'estiment pas de façon explicite la composition corporelle. Elle est exprimée par le ratio entre le poids vif de la vache et un poids attendu, caractérisé par un pourcentage spécifique de lipides, associé au degré de maturité de l'animal. Dans le cas d'Hirooka et al (1998), la composition corporelle n'est pas prise en compte. Les besoins associés à la croissance sont estimés à partir d'un gain de poids quotidien issu de la courbe de référence. La composition corporelle n'intervient dans l'estimation des besoins que via l'énergie contenue dans le gain qui est estimée en fonction du sexe, de la race et du taux de croissance.

Les modèles mécanistes fonctionnant à l'échelle de l'animal partitionnent souvent les besoins de l'animal entre les besoins d'entretien, de gestation, de lactation et de croissance (Bruce et al 1984, Williams et Jenkins 2003a, b). Williams et Jenkins (2003a, b) ont proposé un modèle complet qui estime à partir de l'énergie métabolisable ingérée et des caractéristiques de l'animal : i) l'énergie métabolisable pour l'entretien, ii) l'énergie associée à l'augmentation du métabolisme chez la vache en production par rapport à la vache à l'entretien, notamment pour la digestion et iii) l'énergie retenue dans le gain ainsi que la composition du gain. Il s'agit là d'un modèle mécaniste qui prend en compte les situations tout au long de l'année où l'animal est en perte ou en gain de poids. Leur approche qui consiste à prendre en compte l'augmentation du métabolisme chez l'animal en production permet de réaliser les estimations pour une vache au cours de sa carrière.

\section{3 / Le modèle de la compo- sition du gain : VDR}

\section{1 / Cahier des charges du modèle}

Les caractéristiques du modèle doivent permettre d'estimer les besoins d'une vache en engraissement compte tenu de son âge, de son poids vif, de son état corporel et de l'objectif de croissance fixé par l'éleveur, tous ces facteurs influençant la composition du gain. Son cahier des charges comprend ainsi deux points principaux, il doit être : i) évolutif et pour cela suffisamment mécaniste, mais il doit aussi être suffisamment robuste pour être utilisé dans la pratique ; ii) facilement paramétrable par les futurs utilisateurs dans le cadre d'un usage à des fins de recommandations alimentaires, dans des outils comme INRAtion.

L'étude bibliographique a permis d'identifier les trois approches répondant aux exigences principales de ce cahier des charges :

i) choix d'une démarche factorielle qui consiste à séparer les besoins d'entretien (estimés selon une équation classique) et les besoins pour la croissance ;

ii) association de deux périodes dans le modèle global (croissance en élevage et engraissement) pour prendre en compte des effets intervenant à différents pas de temps ;

iii) utilisation de courbes de référence de poids vif assez facilement ajustables sur des données expérimentales et bonne accessibilité des variables à renseigner par l'utilisateur.

\section{2 / Principes généraux du modèle VDR}

VDR est un modèle qui estime la composition du gain de poids en fonction de la vache et de son objectif de gain, mais également les besoins énergétiques de cette vache au cours de son engraissement. Pour ce deuxième objectif, il additionne besoin d'entretien et besoin du dépôt : 
Besoins $(E N)=[$ BesoinsEntretien $(E M)+$

BesoinsGain(EN) / kg] x kmf ou kL

Dans cette équation, les besoins totaux sont exprimés en Energie Nette (kcal EN), de même que l'énergie pour le gain. Les besoins d'entretien sont exprimés en énergie métabolisable. Les paramètres $\mathrm{kg}$, $\mathrm{kL}$ et $\mathrm{kmf}$ correspondent respectivement aux coefficients moyens de transformation de l'énergie métabolisable en énergie nette pour la croissance tissulaire, pour la lactation et pour le rendement global (entretien et production de viande) retenus par le système UFV INRA (1978).

Les besoins d'entretien sont calculés selon l'équation :

BesoinsEntretien $=\lambda \times 105 \times P V^{0.75}$

105 représente les besoins d'entretien d'une vache tarie exprimés en kcal d'énergie métabolisable par $\mathrm{kg}$ de poids métabolique (Petit 1988). Le paramètre $\lambda$ permet un meilleur ajustement du modèle aux données. Il peut correspondre à des effets d'élevage tels que le mode de stabulation, le niveau alimentaire et l'état d'engraissement.

La composition corporelle et la composition du gain de cette vache sont estimées à partir de celles de vaches de référence comme dans Robelin et Daenicke (1980). L'animal de référence représente une vache «moyenne» en terme de croissance et de composition corporelle.

La figure 2 présente la structure générale du modèle VDR assemblé sur la base de trois sous-ensembles qui estiment :

i) le développement et la composition corporelle de l'animal de référence en croissance puis en engraissement, proche de la vache observée,

ii) la composition du gain théorique de cet animal de référence en engraissement,

iii) la composition et le besoin de la vache observée, calculés à partir de ceux de l'animal de référence.

Le développement avec l'âge de l'animal de référence a été construit grâce aux bases de données disponibles et par expertise. Poids vif et composition corporelle sont calculés en fonction $\mathrm{du}$ format selon un modèle de croissance adapté (cf. 3.3). On détermine ensuite via le squelette de la carcasse $\left(\mathrm{OSCA}_{\text {ref }}\right)$, les poids et composition corporelle en début d'engraissement $\left(\mathrm{PV}_{\text {émacié}}, \mathrm{PVV}_{\text {émacié}}, \mathrm{LIP}_{\text {émacié }}\right)$ et en

Figure 2. Structure d'ensemble du modèle VDR.

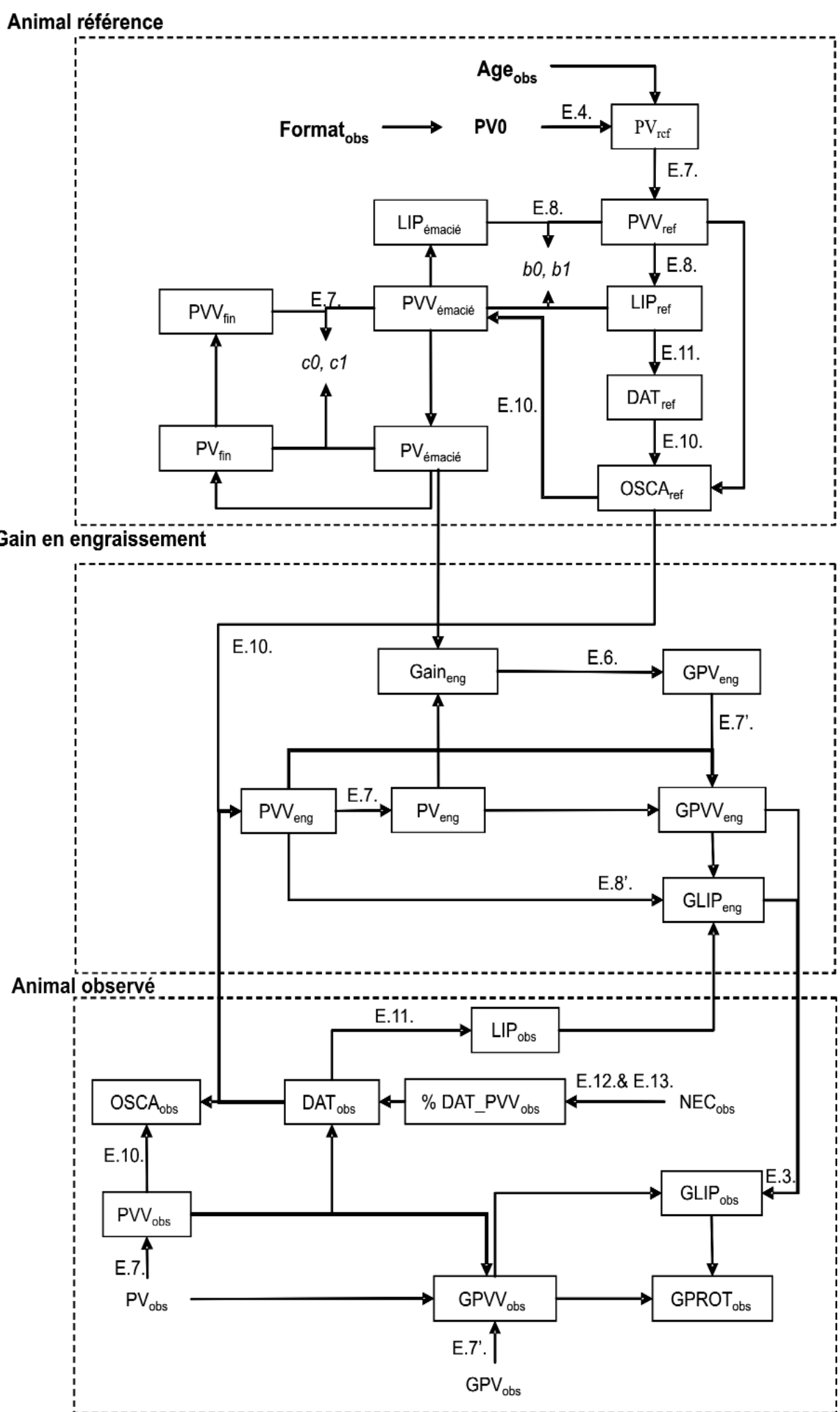

Les paramètres $c 0, c 1, b 0$ et b1 calculés pour l'animal de référence en engraissement servent à l'utilisation des lois d'allométrie. E.2. à E.13. font référence aux équations 2 à 13 dans le texte. L'équation E.7'. correspond à la dérivée de l'équation 7.

fin d'engraissement $\left(\mathrm{PV}_{\text {fin }}\right.$ et $\left.P V V_{\text {fin }}\right)$ (figure 3). Ces deux «points repères» permettent de calculer les coefficients d'allométrie (c0, c1, b0 et b1) qui lui sont associés. Ces coefficients seront ensuite utilisés pour estimer l'évolution de la composition corporelle de la vache observée (cf. 3.4) au cours de son engraissement.

La vache observée est décrite par l'utilisateur (poids, format et note 
Figure 3. Modèle de croissance et d'engraissement pour l'animal référence.

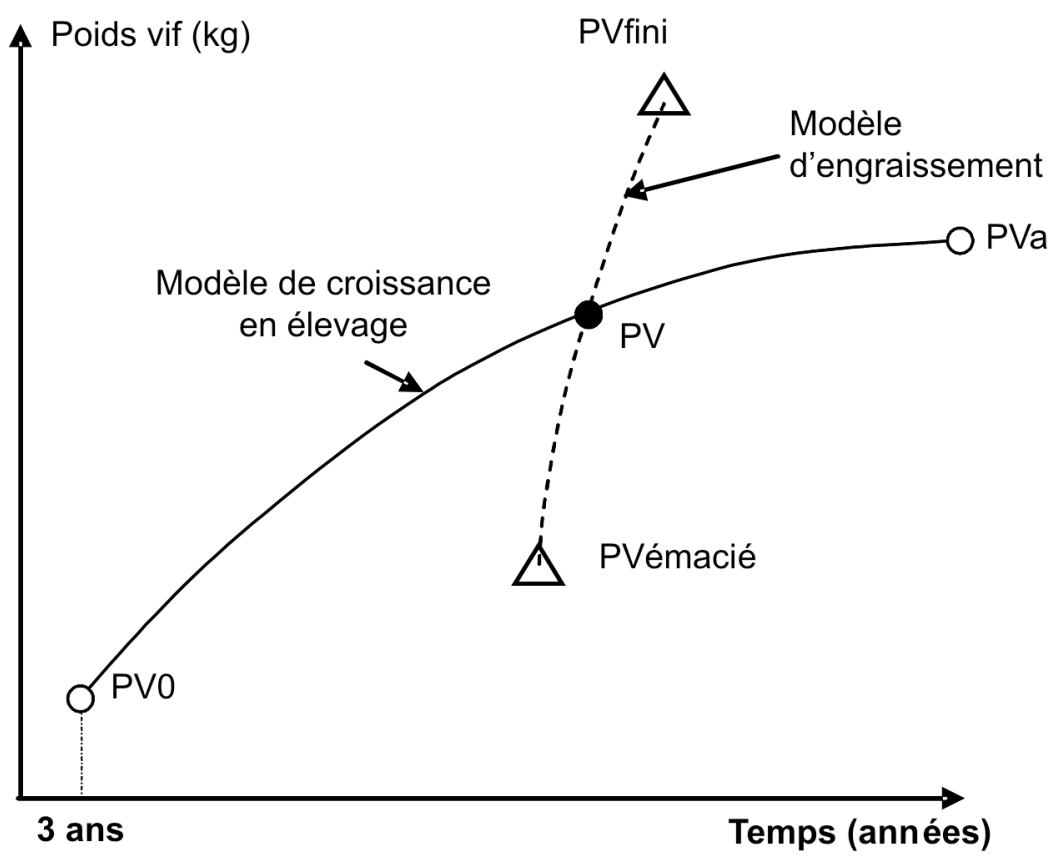

La composition corporelle est estimée aux points o pendant la croissance et aux points $\Delta$ pendant l'engraissement pour calculer les relations d'allométrie. Le point $\bullet$ correspond au point charnière qui permet de faire le lien entre les modèles de croissance et d'engraissement.

d'état) et ces informations permettent de définir sa composition corporelle en termes de poids vif vide, de dépôts adipeux totaux et de squelette OSCA $_{\text {obs }}$ (OSCA $_{\text {ref }}$ doit être peu différent de $\left.\mathrm{OSCA}_{\text {obs }}\right)$.

On calcule également le poids vif vide de l'animal engraissé théorique $\left(\mathrm{PVV}_{\mathrm{eng}}\right)$ qui présente la même valeur de squelette de la carcasse que l'animal référence $\left(\mathrm{OSCA}_{\text {ref }}\right)$ et est caractérisé par la même quantité de dépôts adipeux totaux que la vache observée. Cela permet de déduire un gain théorique cumulé (en $\mathrm{kg}$ ) réalisé par cette vache depuis l'état émacié (Gain ${ }_{\text {eng }}$ ). Grâce à l'équation ajustée avec nos bases de données (cf. 3.3 modèle d'engraissement), on en déduit le gain de poids vif théorique (en kg par jour) de cette vache à l'engrais $\left(\mathrm{GPV}_{\text {eng }}\right)$. Les relations d'allométrie permettent d'estimer la composition de ce gain en lipides $\left(\mathrm{GLIP}_{\text {eng }}\right)$.

En reprenant les principes du modèle de Robelin et Daenicke (1980), l'animal observé diffère peu de l'animal théorique, si bien que l'on peut considérer la relation allométrique (coefficient d'allométrie égal à 1) suivante.

$$
\text { GLip }_{\text {obs }}=\text { GLip }_{\text {eng }} \times \frac{G P V V_{\text {obs }}}{G P V V_{\text {eng }}}
$$

\section{3 / Les modèles de l'animal de référence en croissance et en engraissement : estimation du poids vif et du gain de poids vif}

Le modèle de croissance en élevage permet d'estimer le poids vif de l'animal de référence de même format et de même âge que la vache observée (figure 3). Cette estimation est réalisée en fonction de son poids initial à 3 ans $\mathrm{PV}_{0}$ (en $\mathrm{kg}$ ) et de son âge, selon une équation de Gompertz de la forme :

$P V=P V_{o} \times \exp (b \times(1-\exp (-a \times($ Age-3) $)))$

La variable Age est l'âge de l'animal exprimé en années et $a$ et $b$ sont des paramètres ajustés pour chaque type d'animal. Le poids vif théorique à l'âge adulte, $\mathrm{PVa}$ est égal à $\mathrm{PV}_{0} \times \exp (\mathrm{b})$. Nous avons mis en place une correction du modèle par le format pour tenir compte de la grande variabilité observée. $P V 0$ est recalculé pour chacun des 4 formats définis. Ainsi, on considèrera qu'une vache de race allaitante sera de format très petit, petit, moyen ou grand selon que sa hauteur au garrot sera comprise entre 110 et $120 \mathrm{~cm}, 120$ et $130 \mathrm{~cm}, 130$ et $140 \mathrm{~cm} \mathrm{ou} 140$ et $150 \mathrm{~cm}$, respectivement. De la même façon, pour une vache de race laitière, des hauteurs au garrot comprises entre 115 et $125 \mathrm{~cm}, 125$ et $135 \mathrm{~cm}, 135$ et $145 \mathrm{~cm}$ ou entre 145 et $155 \mathrm{~cm}$ cor- respondront aux formats très petit, petit, moyen et grand, respectivement. Cette estimation du format revient dans la pratique à l'utilisateur, comme il lui revient de déterminer le poids approximatif et la note d'état de son animal. Il convient de signaler qu'il est nécessaire d'avoir une certaine cohérence entre ces trois informations, pour utiliser ce modèle. Cette cohérence sera testée dans l'application du modèle avec INRAtion.

Le modèle d'engraissement estime le gain de poids théorique pendant la finition (figure 4). Nous avons estimé que le poids vif pouvait s'ajuster sur une équation monomoléculaire de la forme :

$$
\begin{aligned}
& P V_{\text {eng }}=P V_{\text {émacié }}+\text { Gain }_{\max } \\
& x(1-\exp (-r \times \text { duree }))
\end{aligned}
$$

Le gain de poids vif théorique quotidien en engraissement (GPV $\left.\mathrm{Gen}_{\text {eng }}\right)$ est obtenu en dérivant cette équation :

$$
G P V_{\text {eng }}=r \times\left(\text { Gain }_{\max }-\text { Gain }_{\text {theo }}\right)
$$

Gain $_{\max }(\mathrm{kg})$ est le gain maximal qui pourrait être réalisé à la fin de l'engraissement, Gain $_{\text {theo }}(\mathrm{kg})$ est la reprise de poids théorique réalisée depuis le début de l'engraissement, duree est la durée d'engraissement (j) et $r$ représente la vitesse à laquelle le gain quotidien diminue jusqu'à être nul.

\section{4 / Estimation de la composi- tion corporelle de l'animal observé}

L'estimation de la composition corporelle correspond à l'estimation du poids vif vide, de la quantité de protéines et de lipides corporels et de la quantité de squelette dans la carcasse. Nous avons utilisé les relations d'allométrie définies par Robelin et Daenicke (1980) pour estimer le poids vif vide et la quantité de lipides corporels :

$$
\begin{gathered}
P V V=P V-C D=c_{\mathrm{o}} \times P V^{c l} \\
L I P=b_{o} \times P V V^{b l}
\end{gathered}
$$

CD (kg) est le contenu digestif et LIP est la quantité de lipides corporels $(\mathrm{kg})$, PVV est le poids vif vide ou masse corporelle, c'est-à-dire (PV - CD). Les équations dérivées correspondantes sont utilisées pour calculer les gains de poids vif vide et les gains de lipides. Les quantités de protéines corporelles (PROT) sont ensuite calculées en fonction de la masse délipidée selon l'équation de Geay et al (1987) : 
Figure 4. Modèle de l'animal en engraissement.

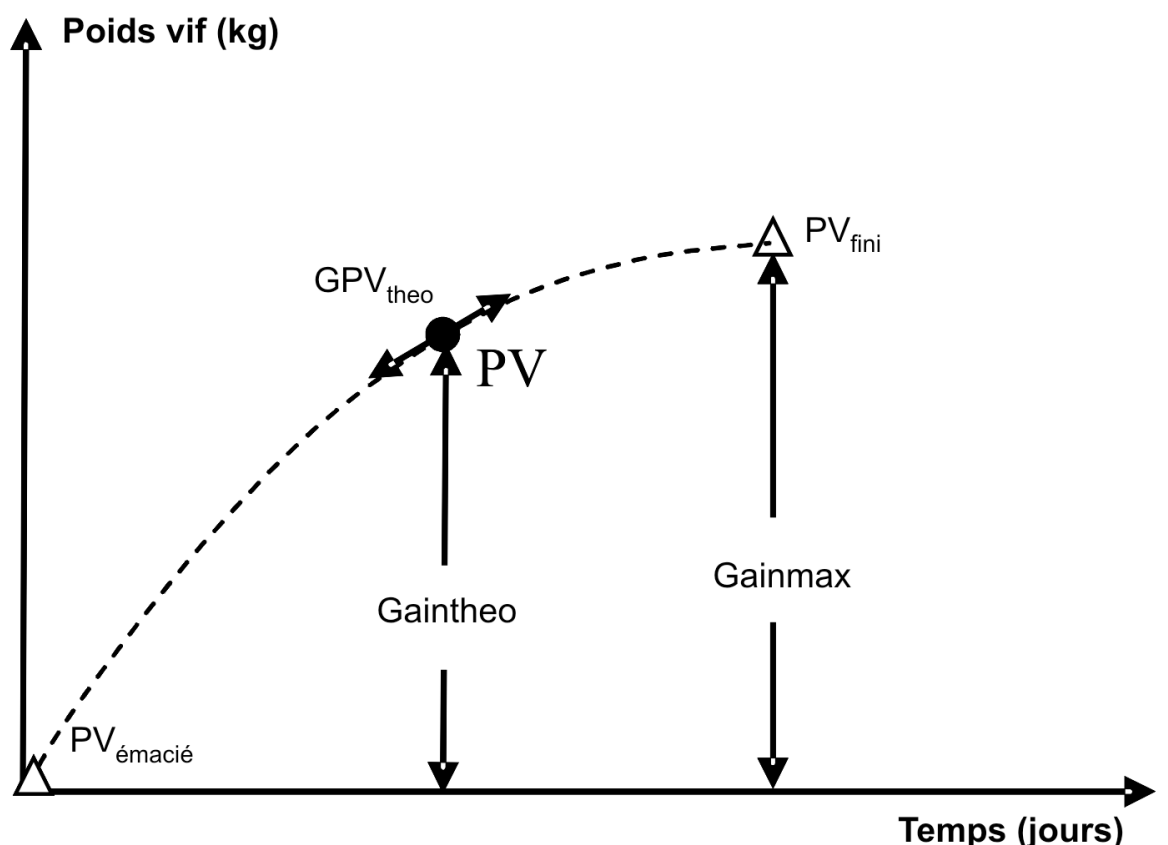

Voir légende de la figure 3.

$$
P R O T=0,1436 \times(P V V-L I P)^{1.0723}
$$

La connaissance de la composition corporelle (contenu digestif et lipides corporels) en deux points (initial à 3 ans, et adulte) a permis d'estimer les paramètres $\mathrm{c} 0, \mathrm{c} 1$, b0 et $\mathrm{b} 1$ du modèle de croissance pour calculer $\mathrm{PVV}_{\text {ref }}$ et LIP $_{\text {ref. }}$

Pour calculer PVV $\mathrm{eng}_{\text {en }}$ et $\mathrm{LIP}_{\text {eng }}$ lors de la phase d'engraissement, les relations d'allométrie ont été utilisées avec des valeurs de coefficients différentes en considérant la composition corporelle en trois points quand la vache est :

i) maigre (PVV émacié et LIP émacié),

ii) en élevage (modèle de croissance : $\mathrm{PVV}_{\text {ref }}$ et $\mathrm{LIP}_{\text {ref }}$ ) et

iii) en fin d'engraissement (finie : $\mathrm{PVV}_{\text {fin }}$ et $\left.\mathrm{LIP}_{\text {fin }}\right)$.

Pour estimer la quantité de squelette dans la carcasse (OSCA), critère descriptif du format, nous avons utilisé les informations disponibles à l'abattoir expérimental pour ajuster une équation de la forme :

$$
\begin{gathered}
P V V=P V V_{\text {moy }}+d \times\left(D A T-D A T_{\text {moy }}\right) \\
+o \times(O S C A-O S C A \text { moy })
\end{gathered}
$$

$\mathrm{PVV}_{\text {moy }}, \mathrm{DAT}_{\text {moy }}$ et $\mathrm{OSCA}_{\text {mọy }}$ sont les valeurs moyennes de poids vif vide, dépôts adipeux totaux et squelette de la carcasse dans la base de données utilisée pour ajuster les paramètres $d$ et $o$ de l'équation. Dans le modèle global, on fait l'hypothèse que i) le squelette de la Limousine (LI) et Holstein (HO). l'équation dans le texte.

\section{4 / Paramétrage et valida- tion de VDR}

VDR a été paramétré par ajustement des équations sur données expérimentales et par expertise. L'application du modèle à des animaux caractérisés par différents âges, formats, PV, GPV, et NEC nous a permis d'examiner son comportement et d'entreprendre sa validation chez des vaches de race Charolaise pour lesquelles nous disposions de plusieurs jeux de données.

\section{1 / Ajustement des équations à partir des données expérimenta- les}

Nous avons réuni 3 types de données pour procéder au paramétrage du modèle chez les vaches de races Charolaise, Limousine et Holstein :

i) des données de poids vif en fonction de l'âge des vaches des unités expérimentales de Theix, des Monts Dore (sites de Laqueuille et Orcival) et de Marcenat qui correspondent à des mesures réalisées en routine sur des animaux «normaux» (Equation 4);

ii) des données de composition corporelle (dépôts adipeux totaux, squelette de la carcasse) issues de l'abattoir expérimental de Theix (Equation 10) ;

iii) des données de cinétique d'engraissement (évolution du poids vif au cours de l'engraissement, Equation 6).

Le tableau 1 présente les équations obtenues par ajustement pour les 3 races. Pour l'équation 6 , nous avons réalisé un ajustement chez les vaches de races à viande en utilisant des données sur vaches Charolaises, et un ajustement chez les vaches de races laitières en utilisant des données sur vaches Holstein (tableau 1). Dans les deux cas, nous avons ajusté un modèle non

Tableau 1. Equations ajustées pour les vaches de réforme de races Charolaise $(\mathrm{CH})$,

\begin{tabular}{|c|c|c|c|}
\hline Race & $\mathbf{N}^{\circ}$ & $\mathbf{n}$ & Equation ajustée \\
\hline \multirow{2}{*}{ Charolaise } & 6 & 143 & $\mathrm{GPV}_{\text {eng }}=0,00779^{*}(187-$ Gaintheo $)$ \\
\cline { 2 - 5 } & 10 & 127 & $\mathrm{PVV}=585+1,9^{*}(\mathrm{DAT}-91)+5,8^{*}($ OSCA-60) \\
\hline \multirow{2}{*}{ Limousine } & 4 & 4780 & $\mathrm{PV}=568^{*} \exp \left(0,18^{*}\left(1-\exp \left(-0,56^{*}(\right.\right.\right.$ Age-3) $\left.\left.)\right)\right)$ \\
\cline { 2 - 5 } & 10 & 111 & $\mathrm{PVV}=530+1,3^{*}(\mathrm{DAT}-77)+3,1^{*}(\mathrm{OSCA}-50)$ \\
\hline \multirow{2}{*}{ Holstein } & 6 & 38 & $\mathrm{GPV}_{\text {eng }}=0,0125^{*}(146-$ Gaintheo $)$ \\
\cline { 2 - 5 } & 10 & 168 & $\mathrm{PVV}=537+1,3^{*}(\mathrm{DAT}-94)+4^{*}($ OSCA-55) \\
\hline
\end{tabular}

$n$ est le nombre d'observations utilisées pour ajuster chaque équation et $\mathrm{N}^{\circ}$ est le numéro de 
Figure 5. Gain de poids vif cumulé au cours de l'engraissement par une vache de race Charolaise en fonction de la durée d'engraissement.

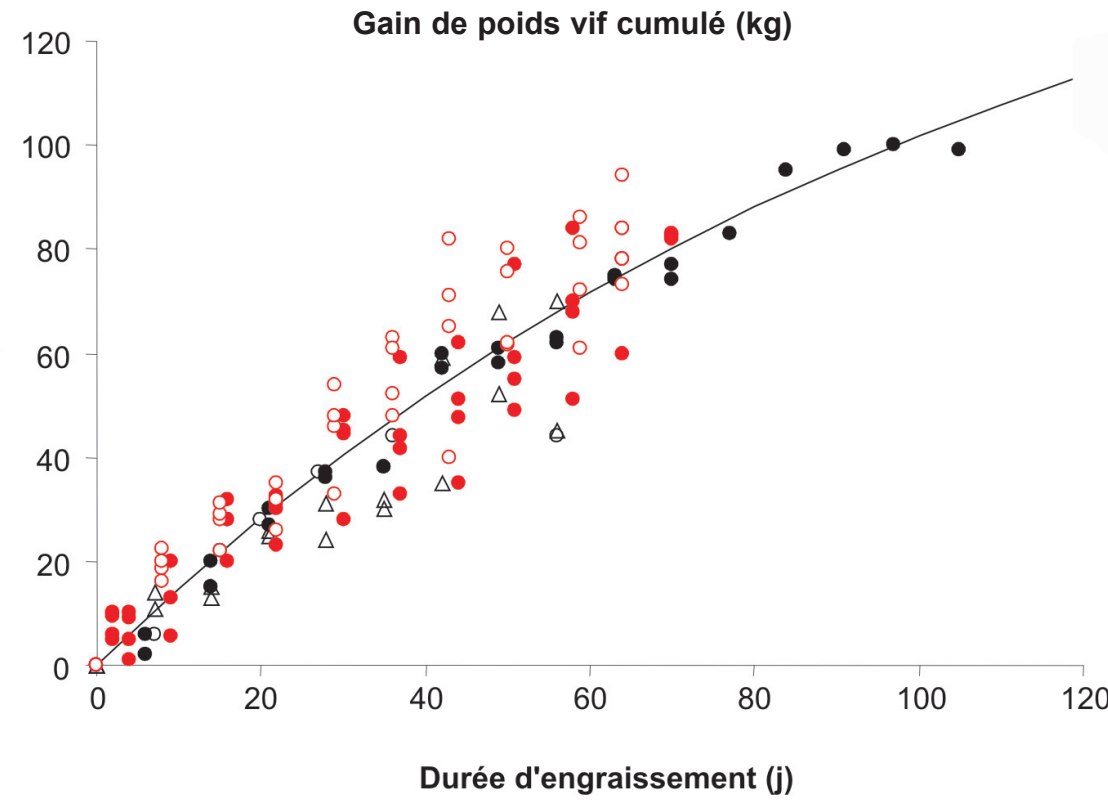

La courbe (-) représente l'équation monomoléculaire ajustée. Les • et $\Delta$ correspondent aux données publiées par Dumont et al (1991) issues d'expérimentations visant respectivement à déterminer l'impact de la durée d'engraissement et de l'âge sur les performances. Les • et o correspondent aux données publiées par Roux et al (1993) issues de deux années d'expérimentation sur l'effet de la suralimentation protéique sur les performances d'engraissement. Les o correspondent aux données issues d'une expérimentation réalisée en 1985 à Laqueuille (non publiée).

linéaire mixte (Proc NLMIXED logiciel SAS) en utilisant le lot expérimental comme effet aléatoire. Les données issues de 5 expérimentations (13 lots) ont permis d'établir la courbe de prise de poids pour les vaches de race Charolaise (figure 5) et les données issues de 2 expérimentations (5 lots) ont permis d'ajuster cette courbe pour des vaches de race Holstein (figure 6).

\section{2 / Validation du modèle chez les vaches de réforme Charolaises}

Nous avons entrepris la validation $\mathrm{du}$ modèle chez les vaches de race Charolaise. Pour cela, nous avons utilisé 31 lots de suivi d'expériences d'engraissement, publiées (Dumont et al 1991, 1997, Roux et al 1993) ou disponibles. Les lots utilisés rassemblent des données moyennes au cours de l'engraissement des quantités d'UFV ingérées par jour, de poids vif, de gain de poids vif, de note d'état corporel et d'âge. La petite base de données ainsi constituée concernait des vaches dont les caractéristiques moyennes sont les suivantes : $5,6 \pm 1,33$ ans, 684 $\pm 48,9 \mathrm{~kg}$ de poids vif, note d'état de $2,6 \pm 0,31$ et $1,3 \pm 0,23 \mathrm{~kg} / \mathrm{j}$ de gain de poids vif.

Le modèle VDR a été utilisé pour prédire la composition moyenne du gain de masse corporelle de chacun des lots, et donc les besoins de production correspondants exprimés en UFV. Pour les comparer aux apports expérimentaux, il faut rajouter l'entretien exprimé comme variable d'ajustement pour minimiser la somme des carrés des en UFV. Ce besoin a été ici utilisé écarts entre valeurs UFV prédites par le modèle et apports UFV expérimentaux. Nous avons ainsi trouvé une valeur qui exprimée en énergie nette est de $103 \mathrm{kcal} \mathrm{EN/kgPV} 0,75,(\lambda=1,4)$. Cette valeur est supérieure à celle retenue pour une vache Charolaise tarie en gestation (105 kcal EM/kgPV0,75, Agabriel et Petit 1987) mais cohérente avec les valeurs retenues pour les autres catégories d'animaux dans le modèle de Robelin et Daenick (1980). Nous l'avons conservée pour la suite en considérant alors les besoins totaux prédits en UFV par VDR.

Nous avons réalisé une évaluation des performances de prédiction en les comparant aux UFV apportées et ingérées par les animaux. Nous avons utilisé pour cela une approche synthétique déjà mise en œuvre par Offner et Sauvant (2004) et Garcia et al (2007b) pour estimer le biais moyen, la pente de la régression des valeurs observées sur les valeurs prédites, le carré moyen de l'erreur résiduelle et le coefficient de détermination de la régression $\left(\mathrm{R}^{2}\right)$. Nous avons également examiné les résidus (prédits moins observés) en fonction des caractéristiques de l'animal (poids vif, gain de poids vif...) afin de mettre en évidence d'éventuels biais dans les estimations. Nous avons également réalisé en parallèle la même évaluation pour les recommandations éditées en 1988 afin d'identifier et de quantifier les améliorations obtenues avec le nouveau modèle.
Figure 6. Gain de poids vif cumulé au cours de l'engraissement par une vache de race Holstein en fonction de la durée d'engraissement.

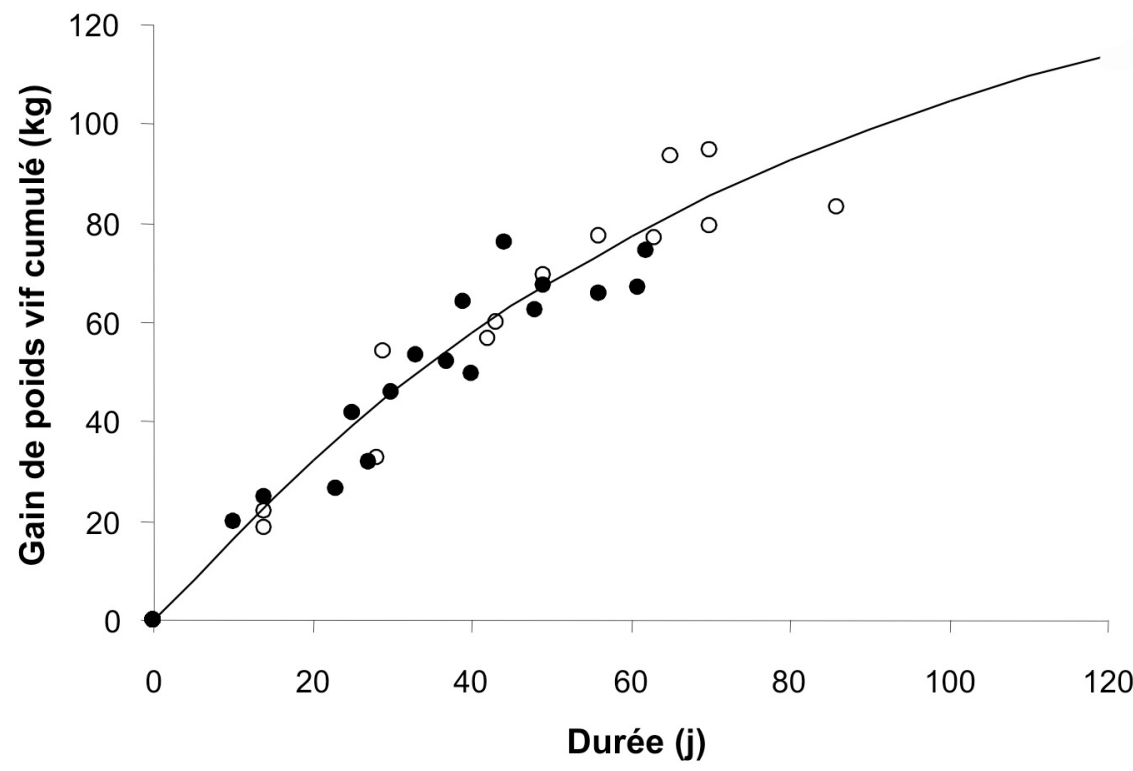

La courbe $(-)$ représente l'équation ajustée à partir des données • de Berge et al (comm. pers.) et des données o de De La Torre et al (2006). 
Figure 7. Comparaison entre les quantités d'UFV ingérées par jour et les besoins UFV prédits par les recommandations $1988(A)$ et par le modèle VDR $(B)$ pour les 31 lots de vaches Charolaises en engraissement.
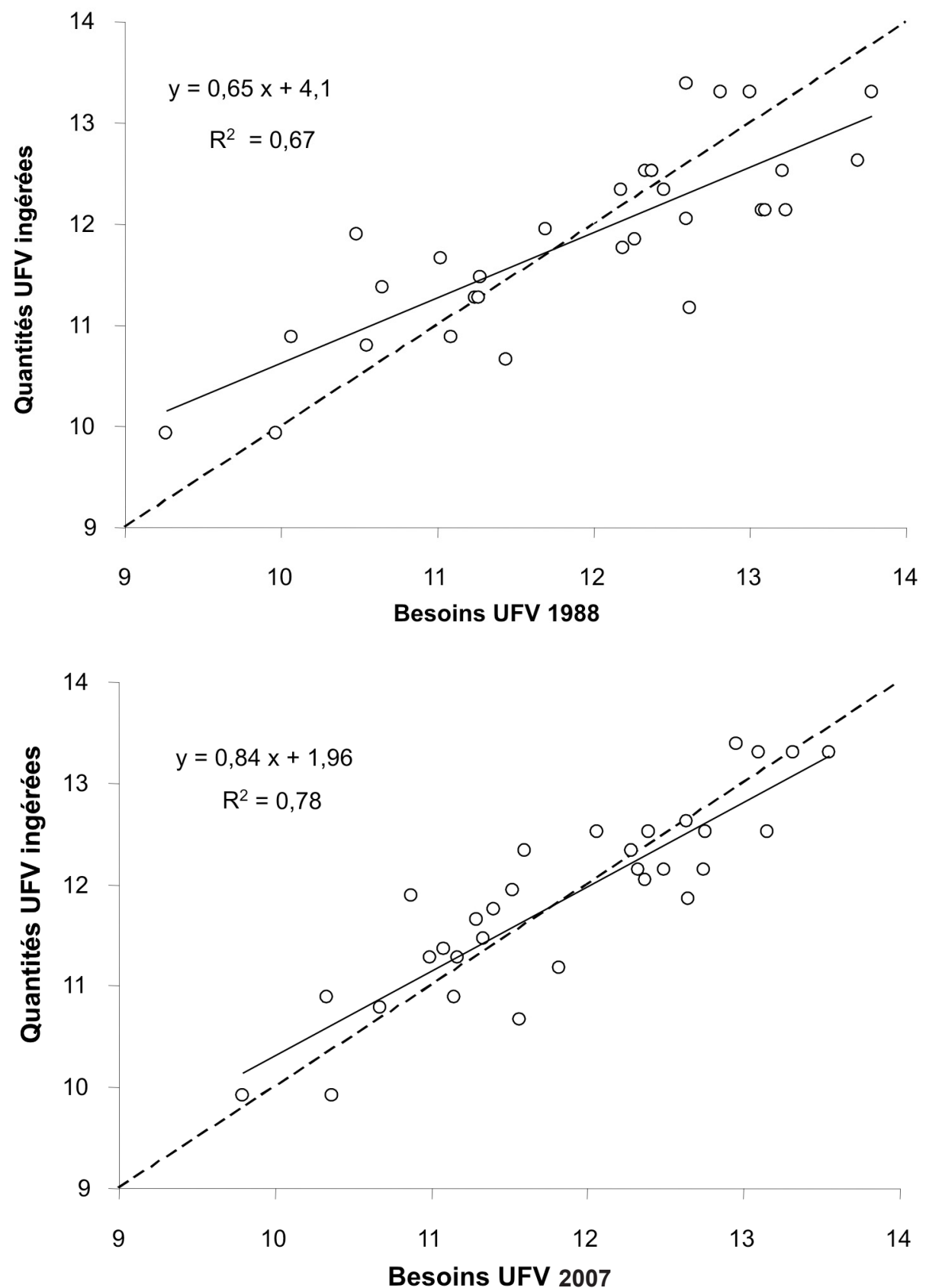

La figure 7 présente les graphes des valeurs UFV observées en fonction des valeurs UFV prédites par les recommandations établies en 1988 (A) et par le modèle VDR (B). Elle suggère visuellement que la relation UFV observés - UFV prédits est meilleure avec le nouveau modèle VDR.

L'évaluation synthétique des deux modes d'estimation des besoins confirme ce résultat :

- le biais moyen obtenu avec VDR est plus faible (- 0,01 versus 0,06$)$;

- la pente de la régression est plus proche de $1(0,84$ et $p=0,055$ versus

La fiabilité des estimations est plus élevée avec VDR qu'avec les recommandations de 1988. De la même manière, l'écart type résiduel de la régression UFV observé $=\mathrm{f}(\mathrm{UFV}$ prédit) est plus faible avec VDR $(0,46)$ qu'avec les recommandations 1988 $(0,67)$ ce qui indique des estimations plus précises. L'examen des résidus a montré que les recommandations 1988 surestiment les besoins des animaux au-delà d'un gain de $1,2 \mathrm{~kg} / \mathrm{j}$. VDR n'induit pas ce biais car la relation entre les résidus et le gain de poids vif au cours de l'engraissement n'est pas significative.

L'examen identique des résidus en fonction des autres variables d'état (note d'état, poids vif, âge...) n'a pas non plus permis de détecter d'autres biais.

Ces résultats demandent à être examinés avec une base de données plus importante et sans réajustement initial du besoin d'entretien, afin d'être sûrs de ne pas surestimer les performances de VDR. Mais le modèle est adapté aux types génétiques et le nombre d'observations de la bibliographie n'est pas pour l'instant suffisant pour entreprendre une validation externe complète sur plusieurs races.

\section{5 / Application aux nouvel- les recommandations ali- mentaires pour les vaches de réforme à l'engrais. Un modèle synthétique de cal- cul des besoins énergétiques}

Le modèle VDR, grâce à la prédiction de la composition du gain, permet de calculer les besoins en UF et protéines déposées des vaches en engraissement connaissant les caractéristiques suivantes : âge, race, format, poids vif, note d'état corporel. L'utilisateur doit ensuite déterminer l'objectif de gain de poids vif de son animal afin d'estimer ses besoins journaliers. D'un point de vue pratique, le modèle VDR sera intégré à la prochaine version d'INRAtion pour proposer cette estimation, et tous les calculs seront masqués. Pour ceux qui n'en disposeraient pas, l'implication de nombreuses équations à différentes échelles de temps rend son utilisation difficile. Aussi pour fournir un outil «papier» facilement utilisable, nous avons conçu une version simplifiée du modèle qui permette de calculer les besoins des vaches grâce à un seul tableau synoptique (tableau 2).
Nous avons pour cela considéré les prévisions UF faites par le modèle complet sur une large gamme de poids d'état et d'âge, et ajusté sur les résultats une équation non linéaire spécifique aux vaches allaitantes (en identifiant la race Charolaise ou Limousine) et une 
Tableau 2. Tableau synthétique de calcul des apports énergétiques (UFV) recommandés des vaches de réforme à l'engrais issues des troupeaux allaitants.

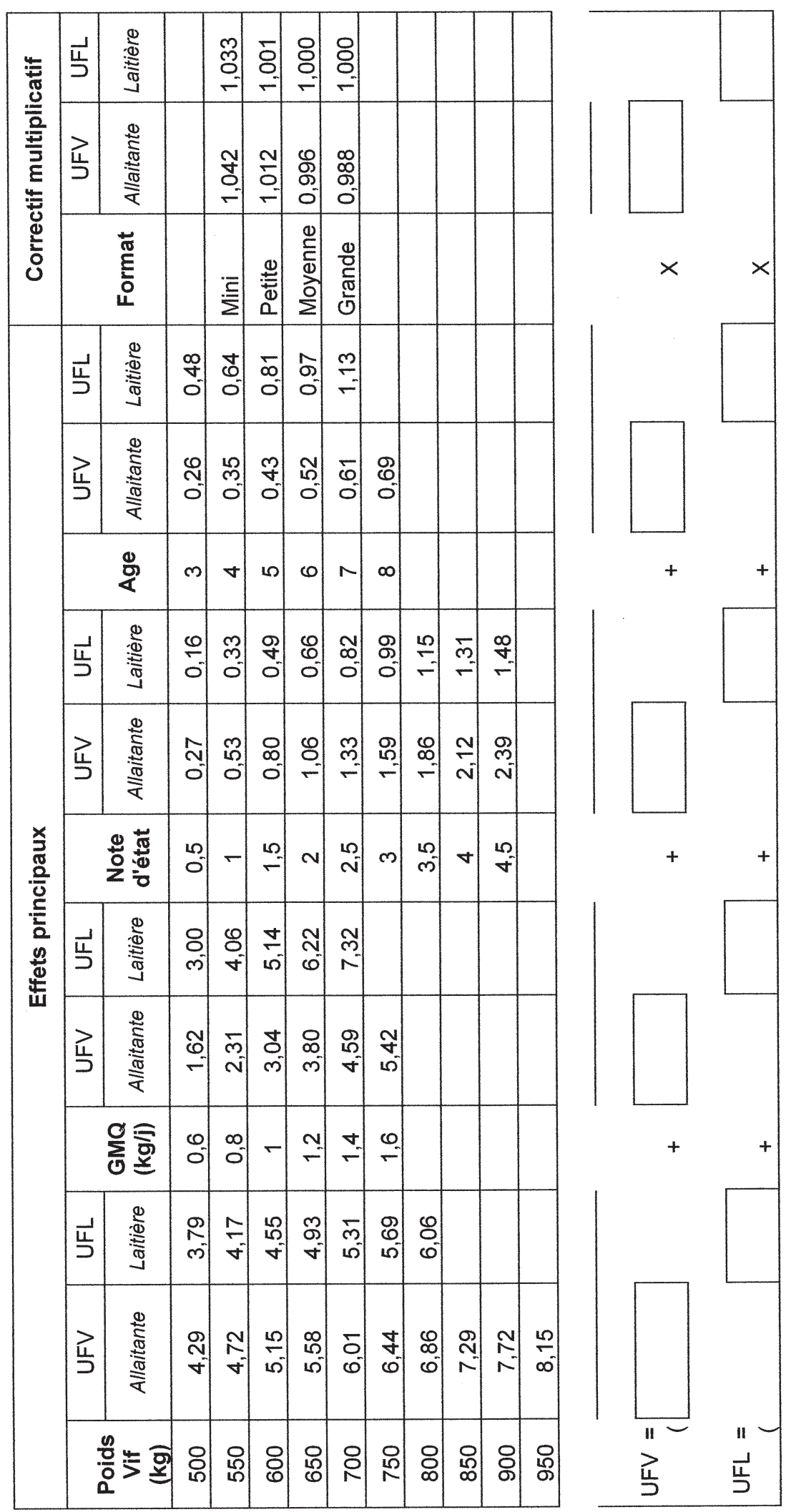

Exemple : pour une vache allaitante moyenne de 5 ans à $800 \mathrm{~kg}$ ayant une note d'état de 2,5 avec un objectif de gain à $1000 \mathrm{~g} / \mathrm{j}$ Besoins $=(6,86+3,04+1,33+0,43) * 0,996=11,61 \mathrm{UFV} / \mathrm{j}$ 
équation spécifique aux vaches laitières (vaches Holstein). Les besoins ont été exprimés en UFV pour les vaches allaitantes et en UFL pour les vaches de race laitière, pour tenir compte du fait que les vaches allaitantes réalisent habituellement un gain de poids en engraissement supérieur à celui des vaches laitières. La forme retenue de l'équation était la suivante :

$$
\begin{aligned}
& \text { Besoins } U F=a \times P V+b \times G P V c \\
& +d \times N E C+e \times \text { Age }
\end{aligned}
$$

où $P V$ est le poids vif de l'animal, $G P V$ son gain de poids vif objectif, $N E C$ est la note d'état corporel et Age est l'âge de l'animal. Dans un deuxième temps à partir des équations ajustées, nous avons établi des équations qui permettent de corriger l'estimation selon le format de l'animal. Nous avons ainsi obtenu les équations 15 et 16 pour les vaches allaitantes et les vaches laitières respectivement :

$$
\begin{gathered}
\text { Besoins }(U F V)=\left(0,0086 \times P V+3,04 \times G P V^{1,23}\right. \\
+0,53 \times N E C+0,09 \times A g e) \\
\times(1,04 \times T P+1,01 \times P+1,00 \times M+0,99 \times G)(15) \\
\text { Besoins }(U F L)=\left(0,0076 \times P V+3,14 \times G P V^{1,05}\right. \\
+0,33 \times N E C+0,09 \times A g e) \\
\times(1,00 \times T P+1,00 \times P+1,00 \times M+1,00 \times G)(16)
\end{gathered}
$$

où $T P, P, M$ et $G$ sont des variables binaires qui prennent la valeur 1 quand le format de l'animal très petit, petit, moyen ou grand respectivement, et 0 sinon. Ces équations nous ont permis de construire le tableau 2 à partir duquel on peut facilement estimer les besoins d'un animal, à l'aide d'une calculatrice éventuellement. Il constitue donc un outil synthétique facile d'accès et applicable sur le terrain.

Nous avons vérifié que ces équations n'induisaient pas de biais majeur dans l'estimation des besoins des animaux par rapport à celle complète faite avec VDR. Pour cela, nous avons comparé les deux estimations dans des gammes de poids d'âge, de format et d'état.

Le biais moyen obtenu (prédiction équation - prédiction VDR) est resté faible $(0,02$ UFV pour les vaches allaitantes et - 0,02 UFV pour les vaches laitières). Dans les deux cas, la régression des valeurs prédites par les équations sur les valeurs prédites par le modèle complet a conduit à des relations satisfaisantes et proches de la bissectrice. L'examen des résidus a néanmoins montré que l'équation simplifiée tend à sous estimer les besoins pour des besoins élevés et qu'elle tend à surestimer les besoins pour les animaux maigres $(\mathrm{NEC}=0,5$ et 1$)$ et les animaux gras $(\mathrm{NEC}=4,5)$. Pour $90 \%$ des estimations, l'erreur réalisée en utilisant l'équation simplifiée par rapport au modèle complet est inférieure à $0,6 \mathrm{UF}$.

En première approche, ces équations donnent donc des résultats satisfaisants mais l'utilisation du tableau simplifié induit des biais, même s'ils restent faibles. Si on cherche une fiabilité et une précision optimale des estimations, il est préférable d'utiliser le modèle complet.

\section{Conclusion et perspectives}

Le modèle VDR est à la base des nouvelles recommandations alimentaires pour les vaches de réforme en finition. Il permet de mieux tenir compte des caractéristiques initiales des animaux, estime la composition du gain de poids défini comme objectif par l'éleveur, puis en déduit les besoins énergétiques. Dans VDR, cette composition du gain de poids est définie de façon originale par couplage de deux modè- les, le premier associé à la phase de croissance en élevage, et le deuxième associé à la phase d'engraissement. Le modèle combine donc deux échelles de temps différentes, l'année pour la carrière de la vache et la journée pour son engraissement.

VDR répond à une approche pragmatique, associant des courbes de croissance de référence et un paramétrage issu des expérimentations menées dans les domaines INRA et de notre expertise. Le modèle établit des estimations des besoins alimentaires correctes pour des vaches de race Charolaise, sans biais associé à l'âge, au format, au gain de poids vif ou encore à la note d'état. Les données sur vaches de réforme de race Limousine ou de race Holstein sont rares et ne nous ont donc pas encore permis d'entreprendre une validation externe du modèle pour ces animaux.

Les estimations réalisées constituent une amélioration par rapport aux recommandations alimentaires de 1988 (Geay et Micol 1988) qui tenaient compte du poids vif et du gain de poids vif de l'animal, mais sans considération de son état d'engraissement ou de son format.

Par ailleurs, la démarche proposée pourrait s'élargir pour servir au développement d'un modèle dynamique de variation de la composition corporelle chez les vaches en production. Dans ce cas, le modèle développé pour la phase de croissance pourrait servir de courbe de référence autour de laquelle le poids et la composition corporelle de la vache fluctueraient au cours des cycles de gestation - lactation. Ce dernier module viendrait alors remplacer le modèle d'engraissement.

\section{Références}

Agabriel J., Lassalas J., 2001. Ingestion de foin par des vaches Charolaises selon leur état d'engraissement. Renc. Rech. Rum., 8, 302.

Agabriel J., Petit M., 1987. Recommandations alimentaires pour les vaches allaitantes. Bull. Tech. C.R.Z.V. Theix, 70, 153-166.

Agabriel J., Giraud J.M., Petit M., 1986. Détermination et utilisation de la note d'état d'engraissement en élevage allaitant. Bull. Tech. C.R.Z.V. Theix, 66, 43-50.

Agabriel J., Garel J.P., Lassalas J., Petit M., 1991. Engraissement des vaches de réforme du troupeau allaitant en conditions de montagne. INRA Prod. Anim., 4 (5), 389-397.
Bastien D., Brouard-Jabet S., 2000. Comment raisonner les limites d'âge dans les cahiers des charges : premières références sur l'effet de l'âge à l'abattage des vaches sur la qualité des carcasses. Renc. Rech. Rum., 7, 269.

Bastien D., Denoyelle C., Tribot Laspiere P., 2002. Age à l'abattage, «suspension pelvienne», pratique de «l'affranchi»: pour une meilleure argumentation des choix techniques en matière de gestion de la tendreté dans les démarches qualité. Renc. Rech. Rum., 9, 251

Bazin S., 1984. Grille de notation de l'état d'engraissement des vaches Pie Noires. I.T.E.B. Publications (Ed), Paris, France, 31p.
Birnie J.W., Agnew R.E., Gordon J., 2000. The influence of body condition on the fasting energy metabolism of nonpregnant, nonlactating dairy cows. J. Dairy Sci., 83, 1217 1223.

Brody S., 1945. Bioenergetics and growth. Reinhold Publishing Corporation (Ed), New York, USA, 1023p.

Bruce J.M., Broadbent P.J., Topps J.H., 1984. A model of the energy system of lactating and pregnant cows. Anim. Prod., 38, 351-362.

Cabaraux J.F., Dotreppe O., Dufrasne I. Clinquart A., Istasse L., Hornick J.L., 2004. Effect of reducing energy supply during the fi- 
nishing of Belgian Blue double-muscled cull cows. Anim. Sci., 79, 469-482

Cabaraux J.F., Dufrasne I., Roux M., Istasse L., Hornick J.L., 2005. La production de viande bovine à partir de femelles de réforme. INRA Prod. Anim., 18 (1), 37-48.

Cabaraux J.F., Hornick J.L., Dufrasne I., Clinquart A., Istasse L., 2003. Engraissement de la femelle de réforme Blanc-Bleu Belge cularde : performances zootechniques, caractéristiques de la carcasse et qualité de la viande. Ann. Med. Vet., 147(6), 423-431.

Chilliard Y., 1987. Revue bibliographique variations quantitatives et métabolisme des lipides dans les tissus adipeux et le foie au cours du cycle gestation-lactation. 2. Chez la brebis et la vache. Reprod. Nutr. Dev., 27 (2A), 327-398.

Chilliard Y., Cisse M., Lefaivre R., Rémond B., 1991. Body composition of dairy cows according to lactation stage, somatotropin treatment, and concentrate supplementation. J. Dairy Sci., 74 (9), 3103-3116.

Chilliard Y., Doreau M., Bocquier F., Lobley G.E., 1995. Digestive and metabolic adaptations of ruminants to variations in food supply. In : Journet M., Grenet E., Farce M.H., Theriez M., Demarquilly C., Recent developments in the nutrition of herbivores. INRA (Ed), Paris, France, 329-360.

De La Torre A., Gruffat D., Durand D., Micol D., Peyron A., Scislowski V., Bauchart D., 2006. Factors influencing proportion and composition of CLA in beef. Meat Sci., 73, 258-268.

Dransfield E., Martin J.F., Bauchart D., Abouelkaram S., Lepetit J., Culioli J., Jurie C., Picard B., 2003. Meat quality and composition of three muscles from French cull cows and young bulls. Anim. Sci., 76(3), 387-399.

Dumont R., Roux M., Agabriel J., Touraille C., Bonnemaire J., Malterre C., Robelin J., 1991. Engraissement des vaches de réforme de race Charolaise - Facteurs de variation des performances zootechniques, de la composition tissulaire des carcasses et de la qualité organoleptique de la viande. INRA Prod. Anim., 4 (4), 271-286.

Dumont R., Roux M., Touraille C., Agabriel J., Micol D., 1997. Engraissement des vaches de réforme de race charolaise - Effet d'un apport de tourteau de lin sur les performances d'engraissement et les propriétés physico-chimiques et sensorielles de la viande. INRA Prod. Anim., 10(2), 163-174.

Faverdin P., Delagarde R., Delaby L., Meschy F., 2007. Alimentation des vaches laitières. In Alimentation des bovins, ovins et caprins. Besoins des animaux. Valeurs des aliments. Editions Quæ (Ed), Paris, France, 23-55.

Fiems L.O., Van Caelenbergh W., Vanacker J.M., De Campeneere S., Seynaeve M., 2005. Prediction of empty body composition of double-muscled beef cows. Livest. Prod. Sci., 92, 249-259.

Garcia F., Agabriel J., Micol D., 2007a. Alimentation des bovins en croissance et à l'engrais. In : Alimentation des bovins, ovins et caprins. Besoins des animaux. Valeurs des aliments. Editions Quæ (Ed), Paris, France, 89-120.

Garcia F., Sainz R.D., Agabriel J., Barioni L.G., Oltjen J.W., 2007b. Comparative analysis of two dynamic mechanistic models of beef cattle growth. Anim. Fd Sci.Technol., accepté.

Geay Y., Micol D., 1988. Alimentation des bovins en croissance et à l'engrais. In :
Jarrige R., Alimentation des bovins, ovins et caprins. INRA (Ed), Paris, France, 213-248.

Geay Y., Micol D., Robelin J., Berge P., Malterre C., 1987. Recommandations alimentaires pour les bovins en croissance et à l'engrais. Bull. Tech. C.R.Z.V. Theix, 70, 173-183.

Hirooka H., Groen A.F., Hillers J., 1998. Developping breeding objectives for beef cattle production. 1. A bio-economic simulation model. Anim. Sci., 66, 607-621.

Hoch T., Picard B., Jurie C., Agabriel J., 2002. Modélisation de l'évolution des caractéristiques des fibres musculaires de bovins. 9èmes Journées des Sciences du Muscle et Technologies de la Viande. Clermont-Ferrand, France, 15-16 octobre 2002. Viandes et Produits Carnés, Hors Série, 121-122.

Hoch T., Pradel P., Agabriel J., 2004. Modélisation de la croissance des bovins : évolution des modèles et applications. INRA Prod. Anim., 17(4), 303-314.

Hoch T., Jurie C., Pradel P., Cassar-Malek I., Jailler R., Picard B., Agabriel J., 2005. Effects of hay quality on intake, growth path, body composition and muscle characteristics of Salers heifers. Anim. Res., 54, 1-16.

Houghton P.L., Lemenager R.P., Hendrix K.S., Moss G.E., Stewart T.S., 1990. Effects of body composition, pre and post partum energy intake and stage of production on energy utilisation by beef cows. J. Anim. Sci., 68, 1447-1456.

INRA, 1978. Alimentation des ruminants. INRA (Ed), Versailles, France, 621p.

NRA, 1988. Alimentation des bovins, ovins et caprins. INRA (Ed), Versailles, France, 478p.

INRA, 2007. Alimentation des bovins, ovins et caprins. Besoins des animaux. Valeurs des aliments. Editions Quæ (Ed), Paris, France, 307p.

Jenkins T.G., Ferrell C.L., 1997. Changes in proportions of empty body depots and constituents for nine breeds of cattle under various feed availabilities. J. Anim. Sci., 75, 95-104.

Malterre C., 1986. Production de viande de vaches de réforme. In : Micol D., Production de viande bovine. INRA (Ed), Paris, France, 247. 269

Malterre C., Jones S.D.M., 1992. Meat production from heifers and cull cows. In : Baker R.D., Micol D., Béranger C. (Eds), Beef cattle production, World Animal Science, Elsevier Scientific Publishing Company, UK, 357-375.

Malterre C., Robelin J., Agabriel J., Bordes P., 1989. Engraissement des vaches de réforme de race Limousine. INRA Prod. Anim., 2 (5), 325 334.

Meffe N. Veillaux C., Bastien D., 2005. Abattage des vaches laitières en lactation: quantification du phénomène et conséquences pour les abattoirs. Renc. Rech. Rum., 12, 375 378.

Ofival, 2006. http://www.office-elevage.fr/ cotations/indexcot.htm

Offner A., Sauvant D., 2004. Comparative evaluation of the Molly, CNCPS and LES rumen models. Anim. Fd Sci. Technol., 112, 107-130.

Ortigues I., Petit M., Agabriel J., 1993. Influence of body condition on maintenance energy requirements of Charolais cows. Anim. Prod., 57, 47-53.

Petit M. 1988. Alimentation des vaches allaitantes. In : Jarrige R., Alimentation des bovins, ovins et caprins. INRA (Ed), Paris, France, 159184

Pierret P., Breuvart A., Eisenzaemmer C., 2002. Variabilité des poids et des conformations de carcasses de femelles bovins Charolaises d'un groupement de producteurs de Bourgogne. Renc. Rech. Rum., 9, 272.

Pierret P., El-Omari A., Dumont R., 2004. Effets des modes de finition des vaches adultes de race Charolaise sur les caractéristiques de qualité des carcasses. Renc. Rech. Rum., 11, 123.

Remond B., Robelin J., Chilliard Y., 1988 Estimation de la teneur en lipides des vaches laitières Pie-Noires par la méthode de notation de l'état d'engraissement, 1(2), 111-114.

Robelin J., Agabriel J., 1986. Estimation de l'état d'engraissement des bovins vivants à partir de la taille des cellules adipeuses. Bull. Tech. C.R.Z.V. Theix, 66, 37-41.

Robelin J., Daenicke R., 1980. Variations of net requirements for cattle growth with liveweight, liveweight gain, breed and sex. Ann. Zootech., 29, 99-118.

Robelin J., Agabriel J., Malterre C., Bonnemaire J., 1990. Changes in body composition of mature dry cows of Holstein, Limousin and Charolais breeds during fattening. I. Skeleton, muscles, fatty tissues and offal. Livest. Prod. Sci., 25(3), 199-215.

Roguet C., Faverdin P., 1999. Modèle dynamique de la lactation des vaches laitières en fonction des apports énergétiques. Renc. Rech. Rum., 6, 156.

Roux M., Dumont R., Agabriel J., Bonnemaire J., Micol D., 1993. Engraissement des vaches de réforme de race Charolaise. Effet d'une suralimentation protéique sur les performances d'engraissement et les caractéristiques physico-chimiques musculaires. INRA Prod. Anim., 6 (3),237-248.

Sanders J.O., Cartwright T.C., 1979a. A general cattle production systems model. I. Structure of the model. Agric. Syst., 4, 217-227.

Sanders J.O., Cartwright T.C., 1979b. A general cattle production systems model. II. Procedures used for simulating animal performance. Agric. Syst., 4, 289-309.

Sauvant D. 1992. La modélisation systémique en nutrition. Reprod. Nutr. Dév., 32, 217-230.

Shemeis A.R., Liboriussen T., Bech Adnersen B., Abdallah O.Y., 1994. Offal components, body fat partition, carcass composition and carcass tissues distribution in Danish Friesian cull cows of different age and body composition. Livest. Prod. Sci., 40, 165-170.

Tess M.W., Kolstad B.W., 2000. Simulation of cow-calf production system in a range environment. I. Model development. J. Anim. Sci., 78, 1159-1169.

Trigg T.E., Topps J.H., 1981. Composition of body weight change during lactation in Hereford British Fresian cows. J. Agric. Sci., 97, 147-157.

Vermorel M., Coulon J.B., Journet M., 1987. Révision du système des unités fourragères (UF). Bull. Tech. C.R.Z.V. Theix, 70, 9-18.

Williams C.B., Jenkins T.G., 1997. Predicting empty body composition and composition of empty body weight changes in mature cattle. Agric. Syst., 53, 1-25.

Williams C.B., Jenkins T.G., 2003a. A dynamic model of metabolizable energy utilization in 
growing and mature cattle. I. Metabolizable energy utilization for maintenance and support metabolism. J. Anim. Sci., 81, 1371-1381.

Williams C.B., Jenkins T.G., 2003b. A dynamic model of metabolizable energy utilization in growing and mature cattle. II. Metabolizable energy utilization for gain. J. Anim. Sci., 81, 1382-1389
Wooten R.A., Roubicek C.B., Marchello J. A., Dryden F.D., Swingle R.S., 1979. Realimentation of cull range cows. 2. Changes in carcass traits. J. Anim. Sci., 48 (4), 823-830.

Wright I.A., Russel A.J.F., 1984a. Partition of fat, body composition and body condition score in mature cows. Anim. Prod., 38, 23-32.
Wright I.A., Russel A.J.F., 1984b. The composition and energy content of empty body weight change in mature cattle. Anim. Prod., 39, $365-$ 369

\section{Résumé}

Les vaches de réforme représentent une part importante de la consommation de viande bovine en France, et une part non négligeable des revenus des éleveurs de bovins laitiers (10 \%) et allaitants (près de $30 \%$ ). Les observations en exploitation font état d'une grande variabilité phénotypique des vaches réformées en termes d'âge, de format, d'état sanitaire, d'état physiologique et d'état d'engraissement. Dans ce contexte, raisonner au mieux l'alimentation des vaches en engraissement est un véritable enjeu pour fournir à l'abattage des vaches bien conformées et d'état d'engraissement satisfaisant.

Les recommandations alimentaires établies en 1988 utilisent le poids vif et le gain de poids vif pour estimer les besoins énergétiques en engraissement. Elles ne permettent pas de prendre en compte l'âge, le format et la note d'état. Dans le cadre de la réactualisation des recommandations alimentaires pour les bovins en croissance et à l'engrais, nous avons développé un nouveau modèle prédictif, appelé VDR, pour estimer les besoins des vaches en engraissement. Cet article recense d'abord brièvement les facteurs influençant les variations de gain de poids et de composition du gain chez les vaches en engraissement et expose les approches de modélisation existantes. Dans un deuxième temps, nous décrivons le principe et les équations du modèle VDR. Ce modèle repose sur une approche originale associant modèle de croissance pendant la carrière de la vache et modèle spécifique de la période d'engraissement. Le paramétrage a été réalisé pour les vaches de races Charolaise, Limousine et Holstein. La validation du modèle entreprise pour des vaches Charolaises montre que VDR explique près de $80 \%$ de la variabilité des besoins énergétiques des vaches. La comparaison entreprise avec les recommandations de 1988 permet de quantifier les améliorations apportées par VDR. Enfin, la construction d'un tableau synthétique simple d'utilisation pour estimer les besoins énergétiques est explicitée en dernière partie.

\section{Abstract}

Update of feeding recommendations for fattening of culled cows. Development of a model for the estimation of the composition of weight gain and associated requirements.

Culled cows account for a large part of beef consumption in France and are a significant proportion of the income for dairy (10\%) and suckling farmers (up to 30\%). On-farm observations highlight large variation in culled cow phenotype in terms of age, frame size, sanitary and physiological state and body condition. Consequently, an important issue for producers of culled cows is the management of feed supply during the finishing period to obtain a satisfactory condition score and conformation for slaughter.

Feeding recommendations established in 1988 consider the live weight and the live weight gain to estimate the energetic requirements for fattening. They do not allow for the age, the frame size or the body condition score. In order to update the feeding recommendations for growing and fattening bovine, we have developed a predictive model, called VDR, in order to estimate the requirements for fattening culled cows. This paper outlines the factors that contribute towards the variations of live weight gain and gain composition for culled cows. The paper goes on to describe the VDR model by summarising the developments from previous modelling approaches and outlining the assumptions and equations of the model. The VDR model combines a growth model for the cow during its productive period and a model for the fattening period. Parameterisation was done for Charolais, Limousine and Holstein cows. Validation using data from Charolais cows shows that VDR explains about $80 \%$ of the variability in energetic requirements. A comparison with the recommendations from 1988 quantifies the improvements obtained with VDR. The last section describes the construction of a table that allows the energy requirements for culled cows to be estimated easily.

GARCIA F., AGABRIEL J., 2007. Recommandations alimentaires pour les vaches de réforme à l'engrais. Développement d'un modèle d'estimation de la composition du gain de poids et des besoins associés. INRA Prod. Anim., 20, 137-150. 
